\title{
AVALIAÇÃO ESTATÍSTICA ENTRE AS ESTIMATIVAS DE PRECIPITAÇÃO DA CONSTELAÇÃO GPM COM SATÉLITE TRMM: UMA ANÁLISE A BACIA HIDROGRÁFICA DO RIO SOLIMÕES
}

\author{
SERRÃO, Edivaldo Afonso de Oliveira- oliveiraserrao@gmail.com \\ Mestrando em Meteorologia- Universidade Federal de Campina Grande \\ WANZELER, Romero Thiago Sobrinho- romero-thiago@hotmail.com \\ Mestrando em Meteorologia - Instituto Nacional de Pesquisas Espaciais
}

SANTOS, Cleber Assis dos- cleber_ufpa@yahoo.com.br

Mestrando em Meteorologia - Universidade Federal de viçosa

GONÇALVES, Layrson de Jesus Menezes- layrsonmenezes@hotmail.com Mestrando em Meteorologia - Instituto Nacional de Pesquisas Espaciais

LIMA, Aline Maria Meiguins de - alinemeiguins@gmail.com

Doutora em Desenvolvimento Sustentável do Trópico Úmido- Universidade Federal do Pará. Programa de Pós-Graduação em Ciências Ambientais

ROCHA, Edson José Paulino da - eprocha@ufpa.br

Doutor em Meteorologia- Universidade Federal do Pará. Programa de Pós-Graduação em Ciências Ambientais

\begin{abstract}
RESUMO: O objetivo deste estudo é analisar estatisticamente as estimativas de precipitação fornecidas pelo algoritmo IMERG da constelação do Global Precipitation Measurement (GPM) e comparar como as estimativas do algoritmo 3B43 do Tropical Rainfall Measuring Mission (TRMM) para a bacia hidrográfica do rio Solimões nos meses de (abril, julho, outubro/2014; janeiro/2015). Além de validar essas estimativas com dados de precipitação em superficie, referente ao banco de dados do INMET. Foram utilizados os coeficientes de: determinação $\left(R^{2}\right)$ para avaliar a proporção da variância do IMERG (y) explicada pelo TRMM (x); correlação de Pearson ( $r$ ), averiguando o grau de correlação entre as estimativas; e de eficiência Nash-Sutcliffe (NS), medindo a concordância entre os valores estimados, sendo (1) admitida perfeita. Além do índice concordância de Willmott (IC) que mede o grau em que a precipitação estimada pelo IMERG do GPM se aproxima da precipitação estimada pelo 3B43 do TRMM. Na avaliação dos erros foi adotado o RMSE (raiz do erro quadrático médio) que é a medida da magnitude média dos erros estimados entre os dois produtos de satélites (3B43 e IMERG). E o MBE (desvio das médias) que indica o quanto o modelo está sendo subestimado $(>0)$ ou superestimado $(0>)$. Foi observado que todos os meses estudados tiveram excelentes índices estatísticos, sobressaindo o mês de outubro que apresentou o melhor desempenho. Porém no verão, existe uma tendência de subestimativa do satélite em relação ao observado pelo INMET. E para o inverno o GPM apresentou características de superestimativa da precipitação medida nas estações meteorológicas do INMET.
\end{abstract}

PALAVRAS-CHAVE: GPM, TRMM, ESTATISTICA.

STATISTICAL EVALUATION BETWEEN THE CONSTELLATION OF PRECIPITATION ESTIMATES WITH GPM SATELLITE TRMM: AN ANALYSIS OF THE RIVER BASIN SOLIMÕES

ABSTRACT: The objective of this study is to statistically analyze the rainfall estimates provided by IMERG Constellation Global Precipitation Measurement algorithm (GPM) and compare how the estimates of the algorithm 3B43 of the Tropical Rainfall Measuring Mission (TRMM) for the basin of the Solimões River in months of (april, july, october/2014, january/2015). In addition to assessing these estimates with rainfall data on surface, referring to the INMET database. The coefficients used were: determination $\left(R^{2}\right)$ for assessing the ratio of the variance of IMERG $(y)$ explained by $\operatorname{TRMM}(x)$; Pearson correlation ( $r$ ), checking the degree of correlation between estimates; the Nash-Sutcliffe efficiency (NS), measuring the correlation between the estimated values, where (1) is a perfect agreement. And the Willmott concordance index (CI) that measure the degree of approach between the precipitation estimated by GPM IMERG and 3B43 TRMM. In the evaluation of the errors was adopted RMSE (root mean square error) which is the measure of the average 
magnitude of errors estimated between the two satellites products (3B43 and IMERG). And the MBE (mean bias error) that indicates how the model is being underestimated $(>0)$ or overestimated $(0>)$. The results showed that every month studied had excellent statistical indices, highlighting the month of October that showed the best performance. But in the summer, there is a satellite underestimation trend compared to that observed by INMET. And for winter GPM showed overestimation characteristics of precipitation measured at weather stations INMET.

KEYWORDS: GPM, TRMM, statistic.

\section{INTRODUÇÃO}

A bacia hidrográfica do rio Solimões é a maior sub bacia ocidental coletora da região Amazônica, estende-se desde a Colômbia (12,15\%), Equador (5,87\%), Peru $(39,09 \%)$ até o Brasil $(42,89 \%)$ com uma área total de $2.214 .327 \mathrm{~km}^{2}$. O rio Solimões e tributários drenam uma vasta região do Peru e muitos de seus afluentes são oriundos da cordilheira dos Andes seguindo para as planícies amazônicas. Após a confluência com o rio Negro, próximo à cidade de Manaus, recebe a denominação de rio Amazonas.

Conhecer a distribuição espacial da precipitação pluvial é de suma importância, pois possibilita avaliar o comportamento desta variável aplicado a modelagem hidrológica de bacias hidrográficas (LI et al., 2010), transporte de poluentes (MORUZZI et al., 2012), dinâmica de rios e estimativa de perdas de solo (CUARTAS et al., 2012), disponibilidade hídrica para cultivares (SANTI et al., 2012) e eventos extremos como enchentes e estiagem (KEENAN et al., 2014). Além de ser fundamental para a gestão dos recursos hídricos, desenvolvimento e planejamento ambiental (FILHO et al., 2013).

A bacia hidrográfica do rio Solimões destaca-se como afluente formador da bacia Amazônica, tendo uma relação direta com a distribuição da precipitação na região andina, além do relacionado com a região centro-oeste da América do Sul (FISCH et al., 1996; BARROS; DOYLE, 1996). As ocorrências de variabilidade climáticas registradas (MARENGO, 2009), principalmente nos últimos 15 anos, demonstram que essa bacia demanda por uma avaliação contínua dos principais mecanismos responsáveis pela sua recarga. Destaca-se que esta região também concentra uma significativa área de biodiversidade florística e faunística da Amazônia (NOBRE et al., 1991; DAVIDSON, et al., 2012; KEENAN, 2014).

As grandes extensões das bacias amazônicas, além da dificuldade de obtenção de dados in situ faz com que a geração de informações indiretas seja necessária para o monitoramento continuado destas áreas. A precipitação pluviométrica é uma variável chave neste controle, pois representa um importante meio de avaliação dos parâmetros de descarga (vazão) e variação de nível (cota) dos principais cursos drenantes, possibilitando analisar as consequências dos períodos de cheias e estiagens na região (BINDLISH; BARROS, 2000; ARAÚJO; GUETTER, 2007; COLLISCHONN et al., 2008; SILVA et al., 2008; CUARTAS et al., 2012).

A precipitação está ligada às nuvens, umidade, circulações atmosféricas e oceânicas via liberação de calor latente e de diluição da salinidade, e ao albedo da superfície, através da modulação da cobertura de neve (TRENBERTH et al., 2007). O conhecimento exato da intensidade de precipitação e acumulação é essencial para a compreensão do ciclo dos fluxos globais de água e do balanço energético do sistema terrestre (HOU et al., 2014). Enquanto a medição de precipitação em um determinado local utilizando instrumentos baseados na superfície é relativamente simples, as medições diretas e uniformes sobre grandes regiões são difíceis, dada a variabilidade espacial e temporal, o tipo e a ocorrência da precipitação (NOBREGA et al., 2008)

Globalmente as redes limitadas de instrumentação superficial e a dificuldade de obtenção de medições in situ sobre os oceanos, significa que uma descrição abrangente da precipitação global só pode ser mensurada a partir do ponto de vista do espaço, ou seja, por satélite (KIDD; LEVIZZANI, 2011). Produtos globais de estimativas 
precipitações por satélite atualmente são baseados em sensores de micro-ondas, calibrados com sensor de infravermelho (IR) além de observações de outras missões de satélites (TAO et al., 2010). Visto isso, uma gama de produtos usando uma variedade de técnicas e metodologias que se fundem (HOU et al., 2014), para melhor estima a precipitação a exemplo do produto 3B43 do TRMM (HUFFMAN et al 2007).

Os sensores IR de satélites podem fornecer estimativas de precipitação inferidas a partir da temperatura do topo das nuvens (SOROOSHIAN et al., 2000), sem obter sua composição vertical (CALHEIROS et al., 2014), com altas resoluções temporais, até 15 minutos de intervalos em algumas plataformas (KULIGOWSKI, 2002.; KIDD et al., 2003). Porém, os sensores de micro-ondas continuam a ser o instrumento de escolha para medir a precipitação (TURK; MILLER, 2005) já que as micro-ondas estão mais relacionadas com a microfísica das partículas precipitantes, por exemplo chuva, neve e granizo (KUBOTA et al., 2007). Outros avanços no desenvolvimento de produtos globais de precipitação requerem medidas de micro-ondas mais precisas e mais frequentes dentro de uma estrutura unificada observacional (JOYCE et al., 2011). Nos últimos dezoito anos desde o lançamento do TRMM (Tropical Rainfall Measuring Mission) e atualmente com o GPM (Global Precipitation Measurement) tem-se aprimorado as formas de estimativa da precipitação, auxiliando em diversas funções para a sociedade.

O satélite TRMM é um projeto em parceria entre a NASA e a Japan Aerospace Exploration Agency (JAXA), e foi lançado em 27 de novembro de 1997 com o objetivo específico de monitorar e estudar a precipitação nos trópicos, além de verificar como a mesma influencia o clima global (KUMMEROW et al., 2000).

O GPM é um programa, desenvolvido pela National Aeronautics and Space Administration (NASA) em conjunto com Japan Aerospace Exploration Agency (JAXA), que dispõe de um consórcio de agências espaciais internacionais, incluindo o Centro Nacional de Estudos Espaciais (CNES), a Organização de Pesquisa Espacial da Índia (ISRO), a Administração Oceânica e Atmosférica Nacional (NOAA), a Organização Europeia para a Exploração de Satélites Meteorológicos (EUMETSAT), e outros que visa monitorar globalmente, por meio de satélites, as precipitações na atmosfera, em alta resolução temporal de até 3 horas entre as observações (HOU et al., 2014). Assim como os satélites TRMM e GPM-Core, outros satélites de outras agências também fazem parte da constelação do GPM, o Indo-francês (Megha-Tropiques), o (GCOM-W1) do Japão, o (MetOp) europeu, e dos Estados Unidos os (DMSP, Poes, Suomi NPP, JPSS).

O emprego destes instrumentos pode ser útil a sociedade, como: a identificação de locais de tempestade, previsão de inundações, monitoramento de água doce, o aviso deslizamento de terra, a previsão de safra, acompanhamento de doenças transmitidas pela água (NESBITT et al., 2000; LAU; ZHOU, 2012), dentre outras. A sensibilidade dos instrumentos também ajuda a melhorar a compreensão das características de precipitação em regiões montanhosas (BINDLISH; BARROS 2000; BHATT; NAKAMURA, 2005).

Visto isso, e ressaltando que o satélite TRMM está em fase final de operação, o objetivo deste estudo é realizar uma análise estatística e avaliar se as estimativas de precipitação mensal fornecidas pelo algoritmo IMERG N3 do GPM pode servir como um bom substituto ao algoritmo 3B43 V7 do TRMM. Onde será avaliado o comportamento da precipitação pluviométrica na bacia hidrográfica do rio Solimões (período de abril de 2014 a janeiro de 2015) em quatro meses (abril, julho, outubro/2014, janeiro/2015) no intuito de observar a estimativa de precipitação em cada estação do ano neste período. Para confirmar se os dados do GPM, está realmente concordando com os dados de precipitação do TRMM, será feita a análise de desempenho dos dados estimados de precipitação do GPM, com os dados obtidos por estações pluviométricas. Uma vez que os dados do TRMM já são calibrados com dados de pluviômetros, além de haver diversos trabalhos correlatos ao desempenho dos TRMM com dados de superfície (KUMMEROW et 
al., 2000; NESBITT et al., 2000; PEREIRA FILHO et al., 2002; NÓBREGA et al., 2008; ROZANTE et al., 2010; YANG; NESBITT, 2014; YONG et al., 2015).

\section{MATERIAIS E MÉTODOS}

A sistemática empregada adotou o conceito de Garcez e Alvarez (1988) que afirmam que bacia hidrográfica é uma área definida e fechada topograficamente num ponto do curso de água, de forma que toda a vazão afluente possa ser medida ou descarregada através desse ponto. A definição dos limites da bacia do rio Solimões (Figura 1) foi realizada a partir da rede hidrográfica nacional, classificada segundo o sistema Otto Pfafstetter, adotando as sub bacias definidas como de "Ordem 2" (Resolução CNRH n. 30/2002).

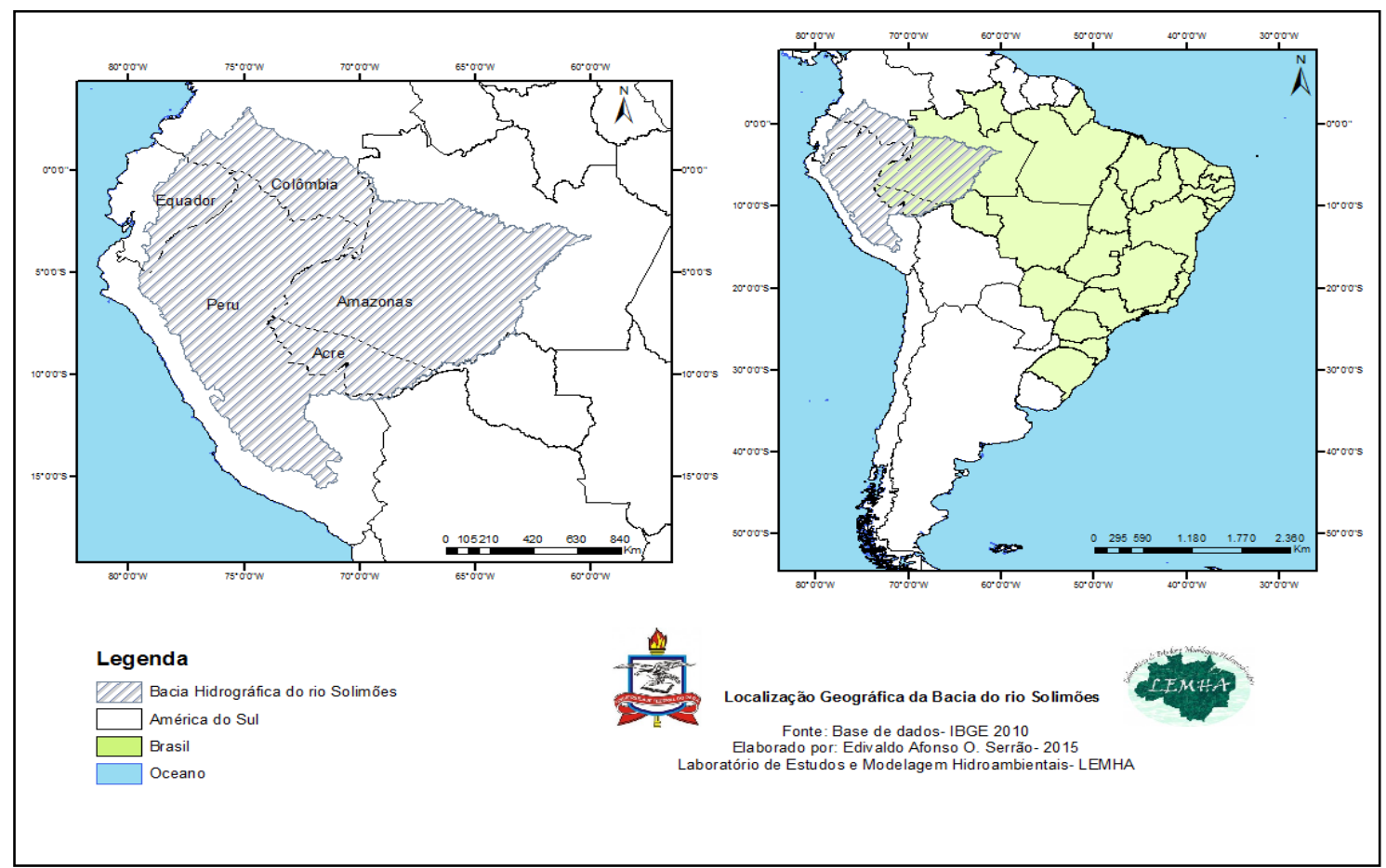

Figura 1 - Localização da área de estudo, bacia hidrográfica do rio Solimões, em toda sua porção territorial (Peru, Equador, Colômbia, e parte do Brasil).

Os dados de precipitação foram obtidos a partir dos dados do produto 3B43 Versão 7 do TRMM e do produto IMERG Nível 3 referente a constelação GPM. No período de abril de 2014 a janeiro de 2015; onde foi avaliada a precipitação em quatro meses: abril, julho, outubro de 2014; e janeiro de 2015. Estes meses marcam de forma bem definida o ciclo hidrológico da região, onde abril representa o período chuvoso, julho o período menos chuvoso, outubro uma parte intermediária do ciclo e janeiro o retorno do período de chuvas na região (Figura 2). 


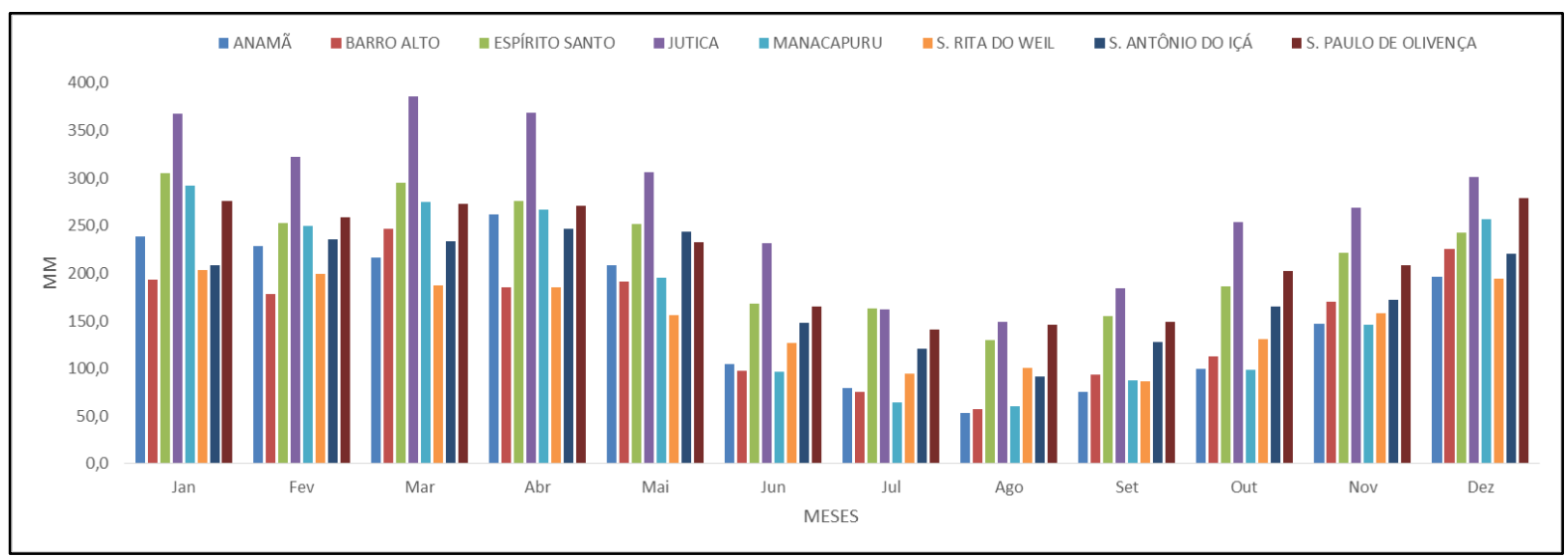

Figura 2 - Distribuição mensal da precipitação pluviométrica, considerando o intervalo temporal de 20 anos (1992 a 2012); em cidades localizadas ao longo do rio Solimões.

O produto 3B43, ou TRMM and Other Sources Rainfall Product (HUFFMAN al., 1995; HUFFMAN et al., 2007) corresponde a series temporais de dados médios mensais de chuva, em milímetros por hora $\left(\mathrm{mm} \mathrm{hr}^{-1}\right)$, calculado a partir dos sensores de Radar de precipitação (Precipitation Radar - PR); Imageador de Micro-Ondas (Microwave Imager- TMI); Escaneador do visível e infravermelho (Visible and Infrared Scanner VIRS), entre outros sensores a bordo do TRMM, assim como dados de pluviômetros em estações provenientes do Global Precipitation Climatological Center (GPCC) produzido pelo serviço meteorológico alemão, World Climate Research Programe (WCRP) e o Sistema de Observação Global do Clima (GCOS). Os dados do produto 3B43 utilizados estão no formato Network Common Data Form (NetCDF) em uma grade de $0.25^{\circ} \mathrm{x}$ $0.25^{\circ}$, recobrindo o globo terrestre entre as latitudes $50^{\circ} \mathrm{N}$ a $50^{\circ} \mathrm{S}$.

Os dados de precipitação do produto Integrated Multi-satellite Retrievals for GPM(IMERG), tem por objetivo combinar as estimativas de precipitação de todos os sensores de micro-ondas da constelação e observações baseadas em infravermelho a partir de satélites geoestacionários (HOU et al., 2014), para obter uma estimativa de precipitação mais precisa. Estes estão em series temporais de dados médios mensais de chuva, em milímetros por hora $\left(\mathrm{mm} \mathrm{hr}^{-1}\right)$, as estimativas de precipitação da constelação GPM são calculados a partir dos instrumentos de Micro-Ondas, do radar de precipitação de dupla frequência (Dual Frequency Precipitation Radar - DPR) e Imageador de Micro-Ondas do GPM (GPM Microwave Imager -GMI). Como os produtos do TRMM 3B42 (COLLISCHONN et al., 2007) e 3B43 (ROZANTE et al., 2010). Os dados do IMERG estão no formato Network Common Data Form (NetCDF) em uma grade de $0.1^{\circ} \times 0.1^{\circ}$, recobrindo 0 globo terrestre entre as latitudes $90^{\circ} \mathrm{N}$ a $90^{\circ} \mathrm{S}$.

O algoritmo de múltiplos satélites do GPM aproveita os algoritmos desenvolvidos ao longo dos últimos 15 anos, que incluem: GPCP (HUFFMAN et al., 2001), PERSIANN (SOROOSHIAN et al., 2000), NRL-Blend (TURK; MILLER, 2005), SCaMPR (KULIGOWSKI, 2002), TMPA (HUFFMAN et al., 2007), CMORPH (JOYCE et al., 2011) o e GSMaP (KUBOTA et al., 2007). Logo, com o IMERG tentou-se criar um produto consistente e calibrado, de modo uniforme em grade, com dados de precipitação global e com mínimo de erros referentes ao observador em superfície. Assim, um satélite pertencente a constelação do GPM, pode corrigir os dados de estimativa de precipitação a partir dos dados de outro satélite da constelação GPM. Uma vez que este também está fazendo medição precipitação pelo globo (HOU et al., 2014).

Na calibração dos dados estimados pelos GPM, foi utilizado dados de precipitação das estações meteorológicas em superfície referentes ao Instituto Nacional de Meteorologia (INMET), uma vez que a bacia hidrográfica do rio Solimões é a maior sub 
bacia coletora a oeste na Amazônia e a dificuldade em obter os dados de precipitação em toda a bacia. Buscou-se analisar seis estações pluviométricas distribuídas entre os dois estados brasileiros (Amazonas e Acre) que fazem parte da bacia do rio Solimões, então foi possível associar a precipitação estimada pelo GPM de latitude e longitude aproximado. (Tabela 1 ).

Tabela 1 - Localização das estações meteorológicas do INMET e pontos de observação do satélite GPM.

\begin{tabular}{c|ccccc}
\multicolumn{1}{c}{ INMET } & \multicolumn{3}{c}{ GPM } \\
\hline ESTAÇÃO & Latitude & Longitude & OID & Latitude & Longitude \\
COARI (AM) & $-4,08$ & $-63,13$ & $\mathbf{3 4 3 7 3}$ & $-4,35$ & $-64,35$ \\
EIRUNEPE (AM) & $-6,66$ & $-69,86$ & $\mathbf{4 6 2 6 4}$ & $-6,55$ & $-69,75$ \\
BEIJAMIN CONSTANT (AM) & $-4,38$ & $-70,03$ & $\mathbf{1 8 1 0 5}$ & $-4,38$ & $-\mathbf{7 0 , 6 5}$ \\
FONTE BOA (AM) & $-2,53$ & $-66,16$ & $\mathbf{3 6 9 0 3}$ & $-2,85$ & $-66,55$ \\
CRUZEIRO DO SUL (AC) & $-7,6$ & $-72,66$ & $\mathbf{4 9 5 5 3}$ & $-7,35$ & $-\mathbf{7 2 , 0 5}$ \\
RIO BRANCO (AC) & 9,96 & $-67,8$ & $\mathbf{2 5 5 2 3}$ & $-9,45$ & $-67,35$
\end{tabular}

Para as análises estatísticas foram utilizados os métodos estatísticos quantitativos de precipitação estimada por satélite recomendadas pelo (IPWG) International Precipitation Working Group, e replicadas por Alves e Vecchia (2011), para isso foi necessário extrair os dados de precipitação dos arquivos em NetCDF, utilizando o software ArcGis 10. Com a ferramenta (Make NetCDF Table View), visualizou-se os dados de precipitação e foram extraídos 18 pontos de observação de precipitação com mesma latitude e longitude para os dois produtos (3B43 e IMERG), cobrindo bacia hidrográfica do rio Solimões. Na cartografia resultante, foi usada a ferramenta (Make NetCDF Raster Layer), possibilitando averiguar a espacialização da precipitação na bacia, e a partir disto pôde-se identificar as regiões de maior e menor distribuição pluvial, no período estudado.

De forma complementar foram calculados o erro médio quadrático (RMSE - root mean square error), o desvio das médias (MBE - Mean Bias Error) o coeficiente de determinação $\left(r^{2}\right)$ o coeficiente de correlação de Pearson ( $r$ ) o coeficiente de eficiência Nash-Sutcliffe (NS) e por fim o índice de concordância (IC), com base em Willmott (1982), Hallak e Filho (2011) e Santos et al. (2011).

$\mathrm{Na}$ tentativa de melhorar a acurácia estatística dos dados, foi utilizado todos os métodos estatísticos do IPWG, para analisar se os dados estimados pelo GPM obtêm um bom desempenho ao comparar com os dados medidos pelo INMET. Adicionalmente foi inserido outro indicativo de erros, com relação entre os dados de precipitação estimados e medidos. O Erro Relativo de Volume Precipitado (ERRV\%), e na análise qualitativa dos dados empregou-se o coeficiente de qualidade (U), segundo Theil (1966).

O RMSE é a medida da magnitude média dos erros estimados entre os dois produtos de satélites (3B43 e IMERG), tem valor sempre positivo e quanto mais próximo de zero melhor a qualidade dos valores estimados entre os satélites (equação 1 ).

$$
R M S E=\sqrt{\frac{1}{n} \sum_{i=1}^{n}\left(E_{T R M M}-E_{G P M}\right)^{2}}
$$

Onde $E_{\text {TRMM }}$ são os valores de precipitação estimados pelo 3B43 do TRMM, e o $E_{G P M}$, são os valores de precipitação estimados pelo IMERG do GPM, e $n$ é o número de pontos de observação dentro da bacia do rio Solimões. Segundo Stone (1993) a desvantagem deste método é que bastam alguns valores discrepantes para que ocorra aumento significativo em sua magnitude. 
O MBE (equação 2) indica o quanto o modelo está sendo subestimado (valor negativo) ou superestimado (valor positivo), os termos ( $E_{T R M M}, E_{G P M}$ e $n$ ) são iguais ao RMSE. O ideal é valor de MBE tendendo a zero. Uma desvantagem apresentada é no cancelamento de um valor positivo por um negativo (STONE, 1993; ULGEN; HEPBASLI, 2009). Ou seja, caso a soma de erros positivos seja igual à soma de erros negativos, o MBE é imparcial (HALLAK; FILHO 2011).

$M B E=\frac{1}{n} \sum_{i=1}^{n}\left(E_{T M M}-E_{G P M}\right)$

$O r^{2}$ (equação 3 ) é definido como a relação que mede a proporção da variação total da variável dependente que é explicada pela variação da variável independente.

$R^{2}=\frac{\sum_{i=1}^{n}\left(\widehat{Y_{I}}-\bar{Y}\right)^{2}}{\sum_{i=1}^{n}\left(Y_{I}-\bar{Y}\right)^{2}}$

A expressão do $r^{2}$ mostra que o coeficiente de determinação é sempre um número positivo entre zero e um, que quanto maior for $0 r^{2}$, melhor será o poder de explicação da reta de regressão. Logo: $\sum_{i=1}^{n}\left(\widehat{Y}_{I}-\bar{Y}\right)^{2}$ corresponde à variação explicada, e $\sum_{i=1}^{n}\left(Y_{I}-\right.$ $\bar{Y})^{2}$ é a variação não explicada.

O coeficiente de correlação de Pearson ( $r$ ) (equação 4) mede o grau da correlação e a direção dessa correlação. Este coeficiente assume valores entre -1 e 1 . Quando 1 significa correlação perfeita positiva entre as duas variáveis, quando -1 significa correlação negativa perfeita entre as duas variáveis, e quando 0 significa que as duas variáveis não dependem linearmente uma da outra.

$R=\frac{\sum(X-\bar{X}) \cdot(Y-\bar{Y})}{\sqrt{\sum(X-\bar{X})^{2} \cdot \sum(Y-\bar{Y})^{2}}}$

O coeficiente de eficiência de Nash-Sutcliffe-NS inicialmente utilizado para avaliar o desempenho de modelos hidrológicos (NASH; SUTCLIFFE, 1970), também eficiente na calibração de estimada de precipitação por satélite e precipitação observada (GUILHON; ROCHA, 2007). Ou ainda para averiguar quanto tuas estimativas ou modelos estão correlacionados e eficientes entre eles (DUAN et al., 1992; KIDD et al., 2003; AONASHI et al., 2009). O coeficiente de eficiência pode variar entre negativo infinito a 1 , sendo o valor 1 indicativo de um perfeito ajuste. Conforme Silva et al. (2008), quando o valor do NS resultar maior que 0,75 , o desempenho do modelo é considerado bom. Para valores de NS entre 0,36 e 0,75, o desempenho é considerado aceitável, enquanto valores de NS inferiores a 0,36 fazem com que o modelo seja julgado como inaceitável. Para este estudo considerou-se como excelentes valores de NS acima de 0,90 onde: $\bar{E}_{T R M M}$, é a estimativa média pelo TRMM

$N S=1-\frac{\sum_{i=1}^{n}\left(E_{G P M}-E_{T R M M}\right)^{2}}{\sum_{i=1}^{n}\left(E_{T R M M}-\bar{E}_{T R M M}\right)^{2}}$

O índice de concordância de Willmott (IC) mede o grau em que a precipitação estimada pelo IMERG do GPM se aproxima da precipitação estimada pelo 3B43 do TRMM. Este índice varia de zero a um, sendo zero, nenhuma concordância e um, concordância perfeita e é dado por:

$I C=1-\frac{\sum_{i=1}^{n}\left(E_{G P M}-E_{T R M M}\right)^{2}}{\sum_{i=1}^{n}\left(\left|E_{G P M}-\bar{E}_{T R M M}\right|+\left|E_{T R M M}-\bar{E}_{T R M M}\right|\right)^{2}}$

Erro Relativo de Volume Precipitado (ERRV), dado em porcentagem, nos remete a quanto os dados medidos estão sendo subestimados $(0<)$ ou superestimados $(>0)$ pelos dados estimados pelo GPM (equação 7). Onde $E_{\mathrm{GPM}}$, É a precipitação estimada e $\mathrm{P}_{\mathrm{INMET}}$, é a precipitação medida nas estações meteorológicas.

$\operatorname{ERRV}(\%)=100 \cdot \frac{\sum_{i=1}^{n} E_{G P M}-P_{I N M E T}}{\sum_{i=1}^{n} P_{I N M E T}}$ 
O coeficiente de $U$ (Theil) (equação 8 ), consiste em uma medida qualitativa de precisão, ao compara os dados estimados com os dados observados (SOUZA; CAMARGO, 2004). Assim quando: $U>1$, o erro da estimativa é maior que o observado, superestimando o real. $U<1,0$ erro da estimativa é menor que o observado, subestimando o real. $U \cong 0$, melhor será o resultado dos dados estimados em relação ao medido.

$U=\sqrt{\frac{\sum_{i=1}^{n}\left(E_{G P M}-P_{I N M E T}\right)^{2}}{\left(\sum_{i=1}^{n}\left(P_{I N M E T}\right)^{2}\right.}}$

\section{RESULTADOS E DISCUSSÕES}

\subsection{Avaliação Estatística das Estimativas de Precipitação (IMERG e 3B43)}

Os resultados estatísticos para o mês de abril/2014, mostrou que as estimativas de precipitação entre os dois produtos estudados (3B43 e IMERG) foram bastante aceitáveis. Onde o coeficiente de Nash-Sutcliffe (NS) foi de 0,99 o coeficiente de determinação $\left(r^{2}\right)$ é de 0,98 e o desvio das médias (MBE) mostrou que o GPM superestimou o TRMM em 0,004 mm. O coeficiente de correlação de Pearson ( $r$ ) e o erro médio quadrático (RMSE) foram de 0,95 e 0,017 respectivamente, ambos bons valores. O índice de concordância de Willmott para o mês de abril/2014 equivale a 1,0 uma perfeita concordância entre as estimativas do 3B43 e IMERG. Observando que para este mês os maiores valores estimados de precipitação foram de 0,55 e 0,58 mm. hr ${ }^{-1} \mathrm{e}$ os menores em 0,0023 e 0,0025 mm. hr ${ }^{-1}$ TRMM e GPM respectivamente representados no Figura 1.

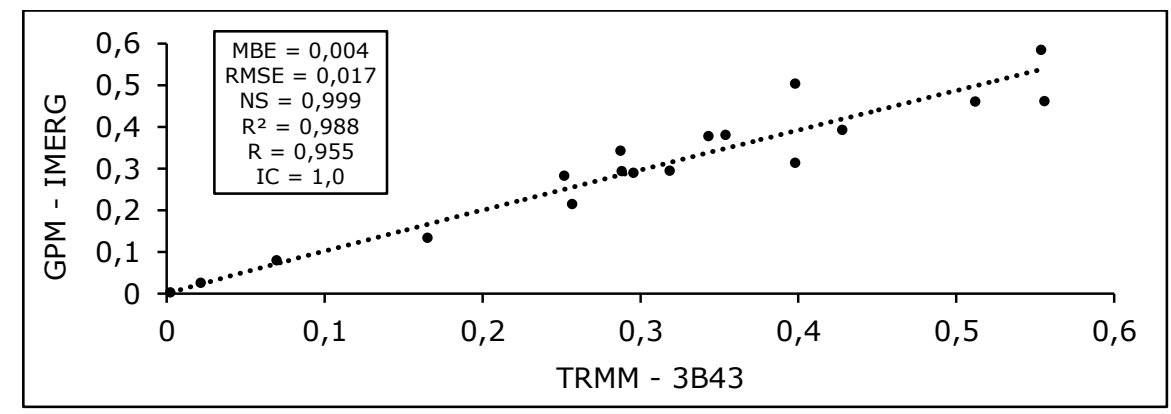

Figura 3 - Resultado estatístico entre (3B43 e IMERG), para o mês de abril/2014.

Os resultados para o mês de julho de 2014 (Figura 2), mostra que as estimativas de precipitações entre os algoritmos 3B43 e IMERG tiveram um decaimento nos coeficientes, mas ainda aceitáveis. Onde o coeficiente de Nash-Sutcliffe (NS) e o coeficiente de determinação $\left(r^{2}\right)$ são da ordem de 0,94 . O desvio das médias (MBE) mostrou que o GPM superestimou o TRMM em $0,025 \mathrm{~mm}$. No coeficiente de Pearson ( $r$ ) houve um acréscimo mínimo em relação ao abril resultando em 0,96 e o erro médio quadrático (RMSE) com magnitude de 0,11 já o índice de concordância de Willmott no mês de julho de 2014 foi equivalente a 0,98 entre as estimativas dos satélites GPM e TRM. Ressaltando que o mês de julho está inserido na estação do inverno, para o Hemisfério Sul (período menos chuvoso na região norte) logo a maioria dos pontos observados não passaram de $0,25 \mathrm{~mm} \cdot \mathrm{hr}^{-1}$, visto isso o maior valor de precipitação estimado foi de $0,40 \mathrm{~mm} \cdot \mathrm{hr}^{-1}$ nos dois algoritmos estudados, e menores estimativas foram de 0,00022 no 3B43e 0,00035 mm.hr-1 no IMERG. 


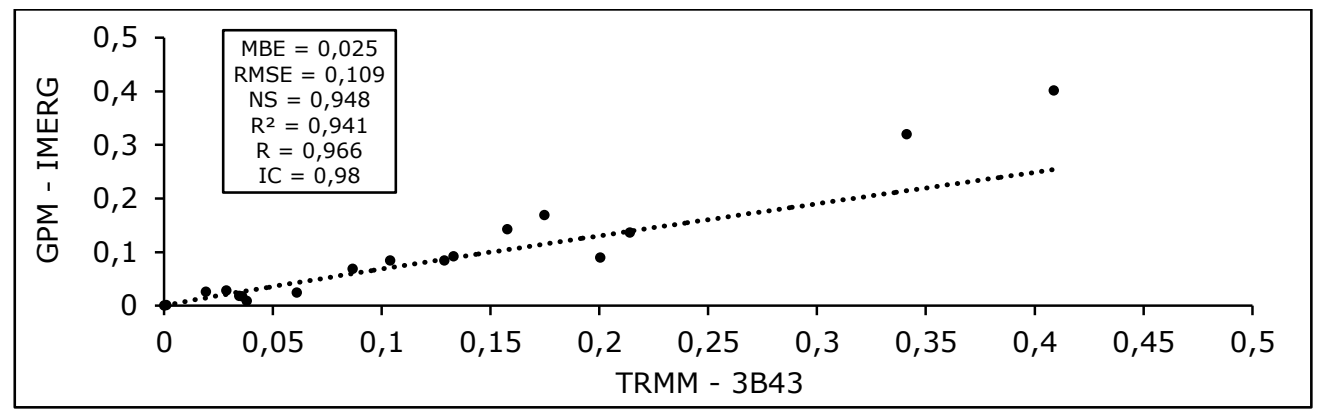

Figura 4 - Resultado estatístico entre (3B43 e IMERG), para o mês de julho/2014.

O mês de outubro de 2014 (Figura 3) demostra que não houve superestimação ou subestimação entre os dois algoritmos estudados com MBE igual a zero, já a magnitude média dos erros (RMSE) foi de 0,001. No coeficiente de Pearson ( $r$ ) houve um decréscimo em relação ao mês de julho resultando em 0,87 ainda considerado uma boa correlação, segundo Alves e Vecchia (2011). Para o coeficiente de determinação $\left(r^{2}\right)$ em outubro obtive-se o valor de 0,97 um acréscimo referente ao mês de julho. Além do coeficiente de determinação o mês de outubro mostrou ainda excelentes valores no coeficiente de Nash-Sutcliffe, bem como no índice de concordância de Willmott sendo 1,0 a magnitude para ambos os indicies estatísticos estudados (SANTOS et al., 2011; ALENCAR et al., 2011). Isto demostra que as estimativas de precipitação entre os algoritmos 3B43 e IMERG estiveram melhor ajustadas neste mês.

Em outubro o Hemisfério Sul vivencia a estação da primavera e ainda encontra-se no período menos chuvoso na região norte, contudo a região sul da bacia hidrográfica do rio Solimões tem um aumento no regime de precipitação, com maior estimativa de precipitação pelo 3B43 com 0,37 mm. hr ${ }^{-1}$ já o algoritmo IMERG estimou $0,34 \mathrm{~mm} \cdot \mathrm{hr}^{-1} \mathrm{a}$ maior concentração entre as estimativas observadas estão entre 0,21 e $0,30 \mathrm{~mm} \cdot \mathrm{hr}^{-1}$.

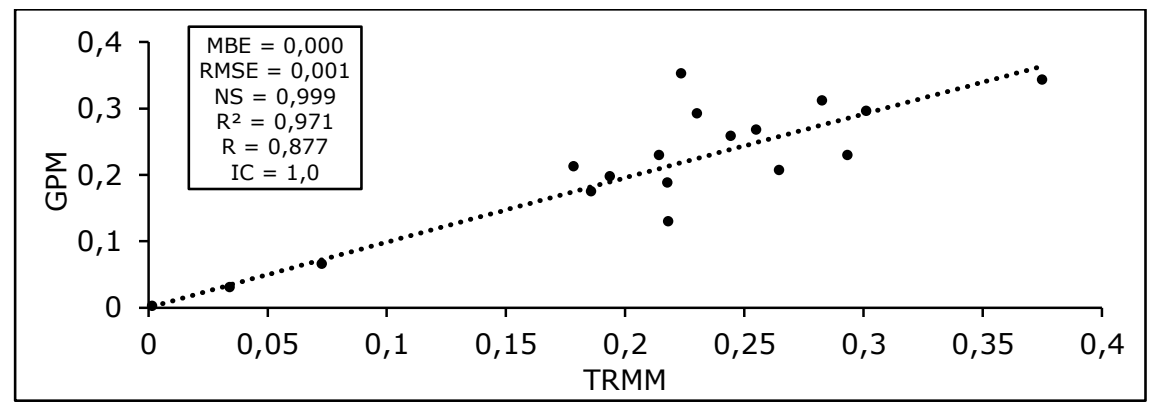

Figura 5 - Resultado estatístico entre (3B43 e IMERG), para o mês de outubro/2014.

O Figura 4 evidencia os resultados para o mês de janeiro de 2015, onde as estimativas de precipitação entre os algoritmos 3B43 e IMERG tive um decaimento no coeficiente de Pearson ( $r$ ) sendo 0,89 seu valor, já o coeficiente de determinação $\left(r^{2}\right)$ teve boa magnitude 0,96 . O desvio das médias (MBE) mostrou que o GPM superestimou o TRMM em 0,007mm, e o erro médio quadrático (RMSE) 0,030 o maior erro quadrático entre o período estudado. O coeficiente de eficiência de Nash-Sutcliffe (NS) equivaleu a 1,0 considerado um excelente ajuste entre os produtos (SILVA et al., 2008; BALTOKOSKI et al., 2010). O índice de concordância de Willmott no mês de janeiro de 2015 obteve valor de 1,0 . Sendo um ajuste perfeito entre as estimativas dos satélites GPM e TRM (HALLAK; FILHO, 2011; ARAÚJO et al., 2011). 


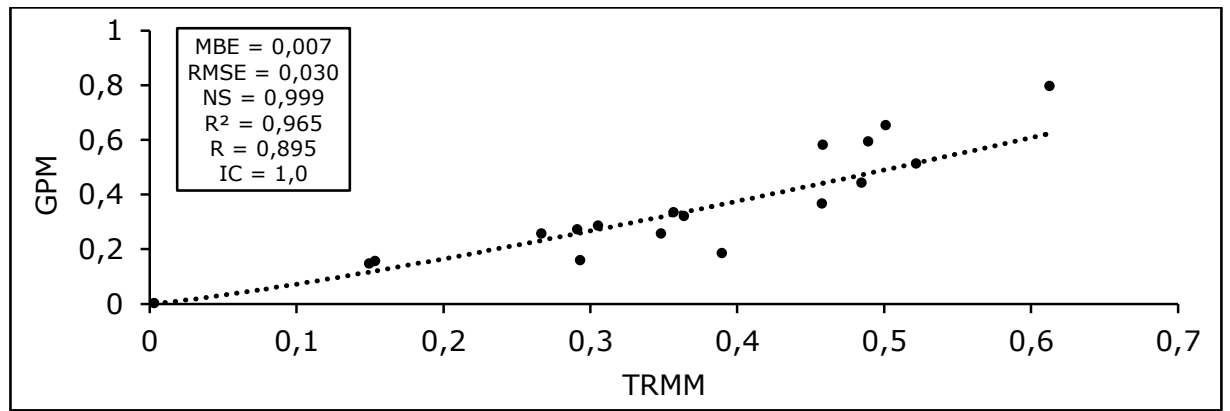

Figura 6 - Resultado estatístico entre (3B43 e IMERG), para o mês de janeiro/2015.

Esses valores de NS e Willmott traduzem uma perfeita relação entre os produtos estudados em janeiro, e este mês estando inserido na estação do verão no Hemisfério Sul (período mais chuvoso na região norte) o maior valor de precipitação estimado foi de $0,79 \mathrm{~mm} \cdot \mathrm{hr}^{-1}$ pelo produto IMERG e $0,61 \mathrm{~mm} \cdot \mathrm{hr}^{-1}$ através do produto 3B43, a concentração da taxa de precipitação ficou entre 0,25 e $0,65 \mathrm{~mm} \mathrm{hr}^{-1}$. Ressaltando que por estar no verão diversos sistemas atmosféricos atuantes na Amazônia contribuíram para esse acréscimo de precipitação.

\subsection{Resultados Estatísticos entre a Precipitação Medida e Estimada (INMET e GPM).}

Os resultados estatísticos referentes aos meses de abril, julho, outubro de 2014 e janeiro de 2015 (Figura 3) demostraram que os dados do estimados pelo GPM são bastante concisos em relação aos dados observados do INMET. Observando o mês de abril (figura 3A), nota-se que o valor máximo de precipitação observado pelo INMET foi próximo de $0,48 \mathrm{~mm} \cdot \mathrm{hr}^{-1}$, enquanto o estimado pelo GPM teve valor máximo de 0,46 $\mathrm{mm} . \mathrm{hr}^{-1}$. Já para o mês de julho (figura 3B) a estimativa ficou em $0,31 \mathrm{~mm} \cdot \mathrm{hr}^{-1}$, e valor observado foi de $0,25 \mathrm{~mm}$. hr ${ }^{-1}$ valores máximos. Já os valores mínimos ficaram em 0,17 e $0,16 \mathrm{~mm} \cdot \mathrm{hr}^{-1}$ medido e estimado, respectivamente.

O mês de outubro (figura $3 \mathrm{C}$ ) apresentou precipitação estimada através do GPM em $0,26 \mathrm{~mm} \cdot \mathrm{hr}^{-1}$, e para a estação meteorológica de referência foi observado 0,28 $\mathrm{mm} \cdot \mathrm{hr}^{-1}$ de precipitação valores máximos de chuva este mês. Em relação ao mínimo de precipitação registrado é de $0,12 \mathrm{~mm} \cdot \mathrm{hr}^{-1}$ e estimado observou-se $0,11 \mathrm{~mm} \cdot \mathrm{hr}^{-1}$. Observando a (figura 3D) demostra $0,25 \mathrm{~mm} \cdot \mathrm{hr}^{-1}$ de precipitação estimada, para 0,22 $\mathrm{mm} . \mathrm{hr}^{-1}$ de precipitação registrada em superficie, valores mínimos. E máximo de chuva observado in situ foi de $0,50 \mathrm{~mm} \cdot \mathrm{hr}^{-1}$, já a precipitação estimada pelo GPM, obteve-se índice de chuva em $0,58 \mathrm{~mm} \cdot \mathrm{hr}^{-1}$.

$\mathrm{Na}$ análise estatística dos coeficientes de dados demostrados na figura 3, mostraram o coeficiente de Nash-Sutcliffe (NS) que melhor se ajustou aos dados observados pelo INMET foi de 0,99 para todos os meses estudados. Isso implica em uma perfeita relação entre os dados estimados pelo GPM. Os coeficientes de determinação $\left(r^{2}\right)$ e Pearson ( $\left.r\right)$ tiveram melhor correlação nos meses de abril, outubro de 2014 e janeiro de 2015. Já o mês de julho de 2014 apresentou valor de (r) igual a 0,86 correlação aceitável.

Para os indicativos de Erros, o (MBE) mostrou que no mês de abril de 2014 foi imparcial, em relação a precipitação estimada e a medida. Mas o GPM superestimou o INMET em 0,007 $\mathrm{mm}$ no mês de janeiro de 2015. E ainda subestimou a precipitação observada in situ nos meses de julho e outubro de 2014. O (RMSE), melhor foi representado no mês de abril de 2014, com erro de 0,001 mm. Enquanto janeiro de 2015 teve o maior erro com 0,046 mm. O (ERRV) apontou os meses das estações de outono e primavera como os de maior subestimativa e superestimativa respectivamente. 
Já o mês de julho de 2014 houve uma subestimativa de $5,7 \%$ da precipitação medida, e janeiro de 2015 a precipitação estimada superestimou em 4,7\% os dados medidos.

Os coeficientes de eficiência, índice de concordância de Willmott e índice qualitativo (U) de Theil, mostraram um ajuste perfeito entre os dados estimados através do GPM se comparados aos dados medidos pelo INMET. Com todos os meses estudados com valores de (IC) em 0,99. O mês de janeiro de 2015 apresentou o melhor desempenho qualitativo $(U)$, de 0,02 . Uma vez que, quanto mais próximo de zero o valor de $U$, melhor os dados estimados estarão em relação aos dados observados.
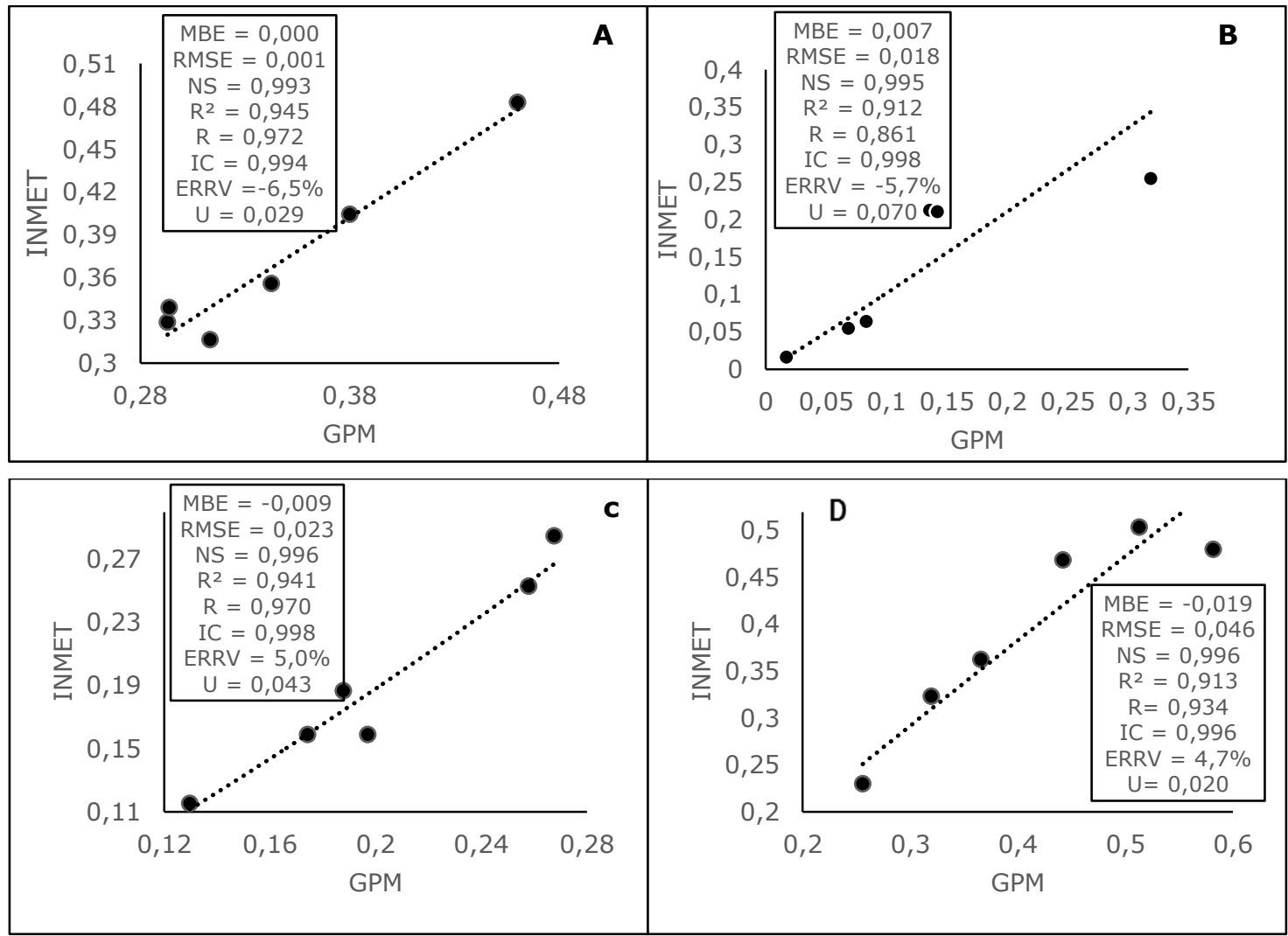

Figura 7 - Resultado estatístico entre (GPM e INMET). (A: referente ao Mês de Abril-2014; B: ao mês de Julho-2014; C: Mês de Outubro-2014 e D: Janeiro de 2015).

\subsection{Analise espacial das Estimativas de Precipitação}

A Figura 8 destaca que o período menos chuvoso foi o que apresentou maior variação segundo os coeficientes MBE, RMSE e NS calculados. Estando o período chuvoso (janeiro e abril) com resultados estatísticos melhores. Como outubro representa uma fase de transição do ciclo, isto é, a recarga hídrica volta a aumenta. O quadro comparativo da distribuição regional da precipitação, observado nas Figuras 9 e 10, mostram um contraste com este resultado onde a espacialização nos meses de janeiro e abril foi mais heterogênea que a dos meses de julho e outubro. As classes de valores (volume precipitado) variaram pouco na comparação 3B43/IMERG, porém sua distribuição, admitindo-se faixas similares, foi diferencial. 


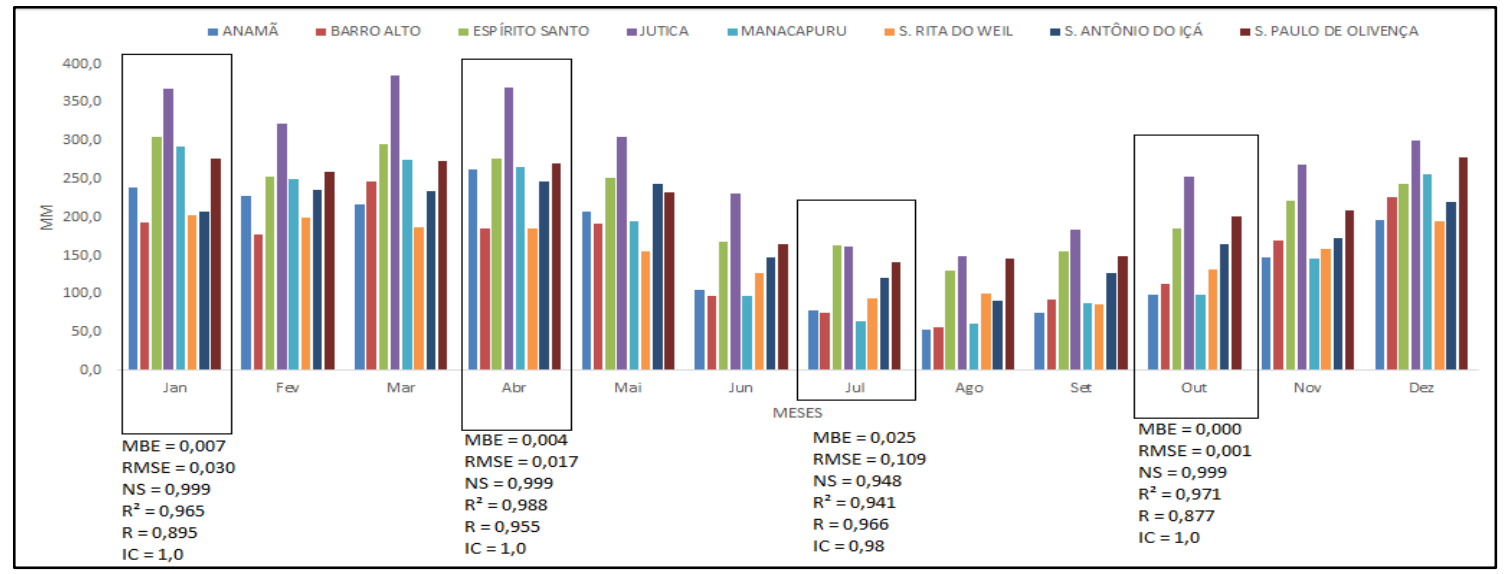

Figura 8 - Análise integrada ao ciclo da bacia do rio Solimões.

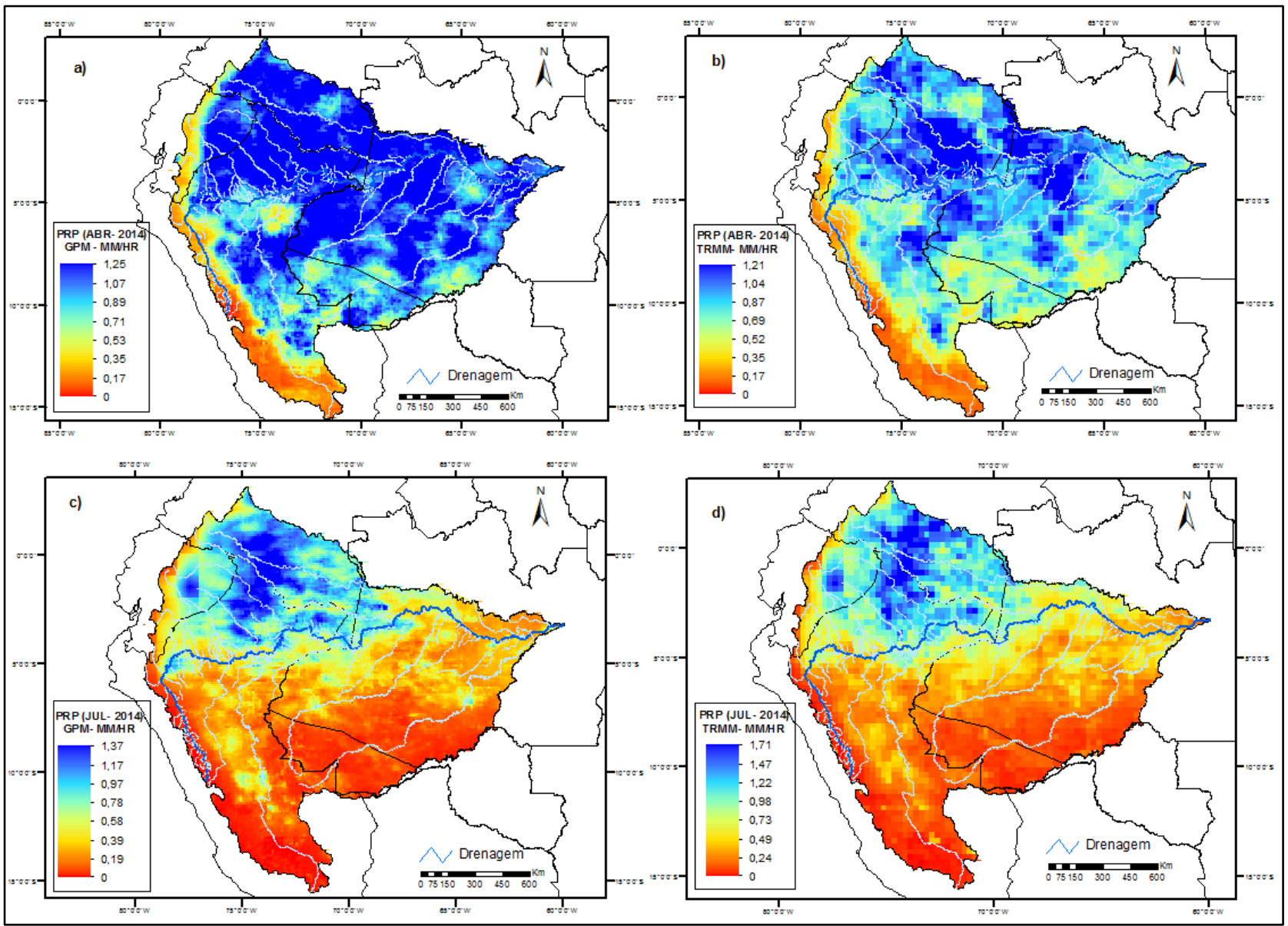

Figura 9 - Espacialização da precipitação da bacia hidrográfica do rio Solimões. (a: precipitação estimada pelo GPM; b: precipitação estimada pelo TRMM, em abril-2014). (c: precipitação estimada pelo GPM; d: precipitação estimada pelo TRMM, em julho-2014). 


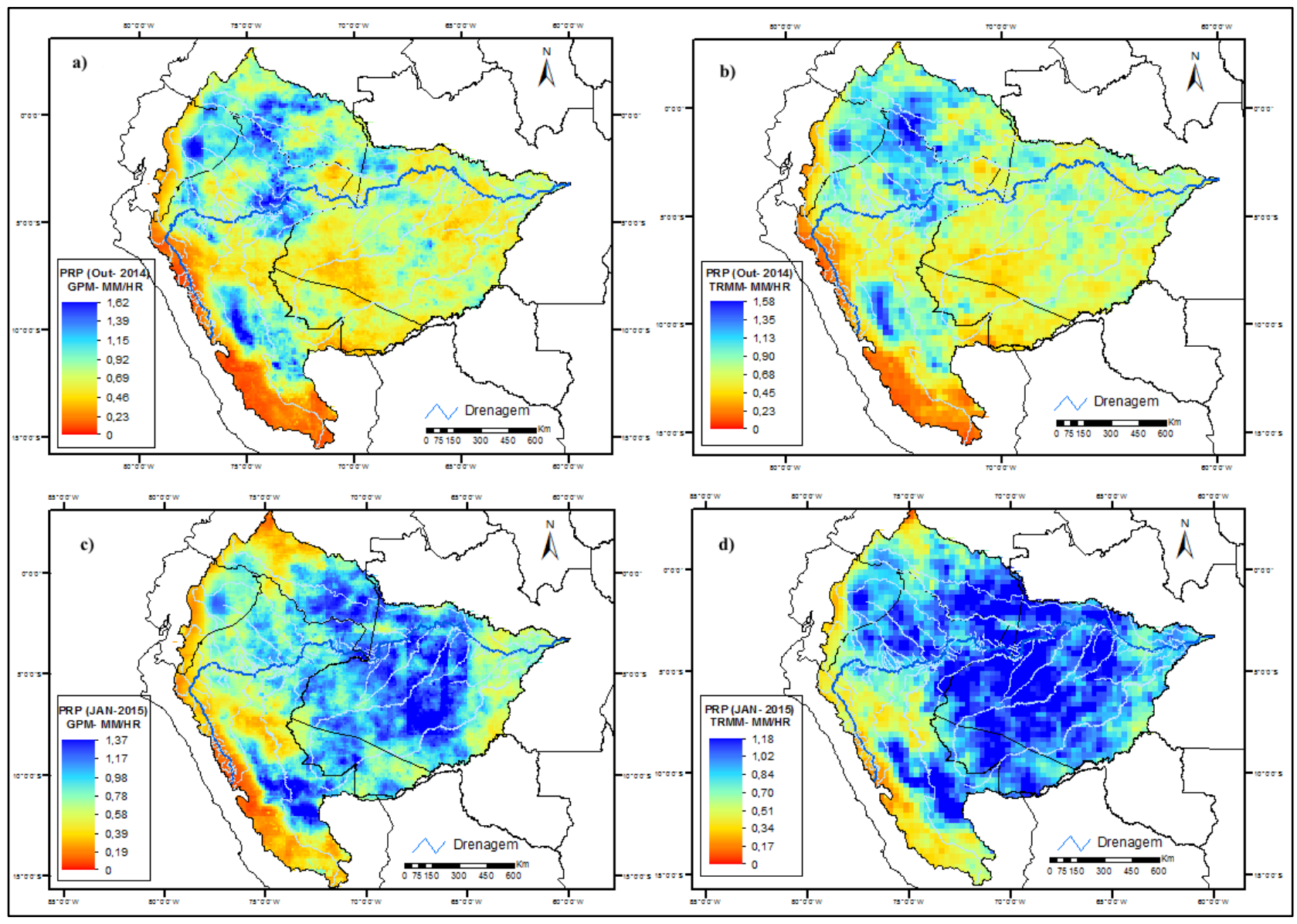

Figura 10 - Espacialização da precipitação da bacia hidrográfica do rio Solimões. (a: precipitação estimada pelo GPM; b: precipitação estimada pelo TRMM, em janeiro-2015). (c: precipitação estimada pelo GPM; d: precipitação estimada pelo TRMM, em outubro-2014).

Como o resultado das Figuras 9 e 10 é produto de interpolação de valores, um dos fatores que pode ser diretamente associado é a resolução do pixel dos satélites GPM (grade de $0.1^{\circ} \times 0.1^{\circ}$ ) e TRMM (grade de $0.25^{\circ} \times 0.25^{\circ}$ ); onde o primeiro tem melhor resolução espacial. Outro fator associado é o de calibração, pois as estimativas de precipitação pelo IMERG não utilizam os dados de pluviômetros como o 3B43, e sim de micro-ondas com sensores de infravermelho (BIN YONG et al., 2015); e estando em um período chuvoso para a região (janeiro e abril), há grande formações de nuvens convectivas e profundas (BARROS; DOYLE, 1996; FISCH et al., 1998; REIBOTA et al., 2010).

Em relação ao mês de julho de 2014 a espacialização da estimativa de precipitação pelo IMERG mostrou-se semelhante ao 3B43, destacando uma maior concentração de chuva a noroeste e a menor em toda porção sul da bacia e a sudoeste. Tal fato reforça a proposta de uma interferência do fator de calibração, pois neste período a menor formação de nuvens pode ter influenciado na resposta mais similar da distribuição espacial da chuva pelos produtos 3B43 e IMERG.

As imagens obtidas por meio dos sensores infravermelhos são compostas de energia radiante que pode ser convertida em temperatura de brilho (por meio da lei de Stefan Boltzmann) (ARAÚJO; GUETTER, 2007) e usadas para inferir alturas de topo das nuvens; onde quanto mais baixas temperaturas de brilho mais altos os topos de nuvem, resultando em grande espessura da nuvem e alta probabilidade de chuva (MAIDMENT, 1993). A relação entre a temperatura de brilho e a chuva é indireta, Carvalho et al. (2005) afirmam que não é possível discriminar a parte convectiva do sistema, da parte 
estratiforme ou nuvens Cirrus, que são nuvens muito frias, porém que não precipitam. Ou seja, medidas de infravermelho tem a desvantagem de não averiguar o conteúdo água completo em toda a estrutura da nuvem, apenas do top, onde há bastante hidrometeoros sólidos havendo discrepâncias nos resultados finais das estimativas.

A avaliação da resposta do TRMM foi testada na bacia do Paraguai (COLLISCHONN, et. al., 2007), na Amazônia Oriental (NÓBREGA, et. al., 2008) bem como, sobre toda a América do Sul (ROZANTE et al., 2010) e os resultados obtidos indicam que este tende a superestimar a precipitação. Yang e Nesbitt (2014) estudaram as estimativas de chuvas observadas pelo radar do TRRM (PR) e mostrou que os algoritmos do TRMM (3B42, 3B43 etc.) tendem a superestimar a precipitação em algumas regiões e subestimar em outras.

Essas variações dependem não só dos instrumentos a bordo do satélite, mas também se dá ao tipo de precipitação predominante do local (HAMED ASHOURI et al., 2015; ZHOU et al., 2015; ZULUAGA; JUNIOR, 2015). Em relação à precipitação TRMM radar (PR), o radar DPR (GPM) é mais sensível às taxas de chuva fraca e queda de neve, além de estar mais associado com a microfísica da precipitação (HOU et al., 2014; KENNETH et al., 2015; MARTINI et al., 2015; TOSHI et al., 2015; ). Pereira Filho et al. (2002) compararam a precipitação derivada do TRMM (sensor TMI), com estimativas de chuva a partir de radar Doppler banda-S e de uma rede de pluviômetros em São Paulo, tendo observado que nenhum dos três instrumentos são efetivos na representação da alta variabilidade espacial e temporal da precipitação, quando analisados separadamente; Huffman et al. (2007) e Yang e Nesbitt (2014) recomendam técnicas estatísticas para integrar os três instrumentos.

\subsection{Perfil do Comportamento da Precipitação na Bacia do rio Solimões}

O observado pela análise integrada da distribuição espacial das Figuras 9 e 10 é que a bacia do rio Solimões, no período analisado, apresentou um comportamento marcado pela presença constante dos maiores índices de chuva a noroeste e menores em toda a faixa ocidental (andina). Seu formato aproximadamente triangular, favorece a variabilidade tanto ao longo do eixo E-W, mais marcante na porção centro-oriental, e $\mathrm{N}-\mathrm{S}$ ao longo do seu eixo centro-ocidental.

Um fator que contribui para a precipitação no noroeste da bacia do rio Solimões está associado à condensação de ar úmido transportado pelos ventos alísios, que sofrem levantamento orográfico próximo aos Andes (NOBRE et al., 1991; DA ROCHA et al, 2009). Os Complexos Convectivos de Mesoescala tropicais (CCMs tropicais) e Sistemas Convectivos de Mesoescala (SCM) são outros sistemas que contribuem para a precipitação na região noroeste da Amazônia (NIETO FERREIRA et al., 2003; SALIO et al., 2007).

Na primavera, segundo Kousky (1985), já é notável a formação ou presença do anticiclone na alta troposfera sobre a América do Sul. A alta começa a ganhar intensidade com a intensificação da convecção na Amazônia, mas é no verão que atinge seu maior desenvolvimento e é denominada como Alta da Bolívia (REBOITA et al., 2010). No verão, para o hemisfério Sul, diversos sistemas precipitantes interagem ainda mais com a convecção local da Amazônia e dão início a um novo ciclo abundante de precipitação na bacia hidrográfica do rio Solimões (SERRÃO et al., 2015):

Destacam-se como processo na Amazônia que favorecem a ocorrência de chuvas intensas: os movimentos convergentes associados à convecção e ao aquecimento proveniente da liberação de calor latente por condensação concomitante com a umidade transportada do Atlântico Tropical pelos alísios para Amazônia promovem a ascensão do ar (MARENGO et al., 2004); a intensa atividade convectiva que favorece o desenvolvimento de um anticiclone em altos níveis (Alta da Bolívia) (SILVA DIAS et al., 
1983; DEMARIA, 1985; FIGUEROA et al., 1995); principalmente na porção norte da bacia Amazônica é observada a influência da Zona de Convergência Intertropical (ZCIT), que nesta época está mais ao sul do equador; e a sul e a sudeste a precipitação é organizada de forma dominante pela Zona de Convergência do Atlântico Sul (ZCAS) (FISCH et al., 1998; SALIO et al., 2007; MARENGO, 2009; ALCÂNTARA, 2011).

\section{CONSIDERAÇÕES FINAIS}

A distribuição da precipitação pluviométrica na bacia do rio Solimões analisada por meio dos sensores TRMM e GPM mostrou os melhores índices de eficiência de NashSutcliffe e Willmott para os meses de abril, outubro de 2014 e janeiro de 2015; os valores obtidos podem ser classificados como excelentes, com menores erros e superestimação mínima entre os dois produtos estudados. Julho de 2014 foi o mês com os menores índices de eficiência, mas não menos expressivos.

$\mathrm{Na}$ avaliação dos dados de precipitação estimado pelo GPM, é perceptível que este está melhor relacionado com a precipitação nos meses de estações de transição (outono e primavera). Já no verão, existe uma tendência de subestimativa do satélite em relação ao observado pelo INMET. E para o inverno o GPM apresentou características de superestimativa da precipitação medida nas estações meteorológicas do INMET. Porém, todos os testes aplicados demostraram que os dados estimados pelo GPM estão em conformidade com os dados de superficie e também com os dados do satélite TRMM.

Observou-se ainda que o produto 3B43 tem a desvantagem da resolução espaçotemporal, quando comparado ao IMERG; porém, apresenta como validação a precipitação pluviométrica registrada in situ, o que torna o dado mais próximo do quantitativo de chuva que pode recarregar hidricamente a bacia. É indicado que hajam mais estudos sobre o produto IMERG para diferentes regiões e metodologias, como foi realizado com o TRMM, para que se possa aprender mais sobre este algoritmo que pode contribuir para os indicativos de precipitação, especialmente na Amazônia.

A combinação das leituras dos múltiplos satélites para todo o globo permite avaliar melhor a precipitação no espaço-tempo, entender a estrutura tridimensional de tempestades, estudar a microfísica da precipitação e assim melhorar as estimativas de chuva. O produto IMERG pode ser um bom substituto ao algoritmo 3B43 do TRMM no estudo do comportamento da precipitação pluviométrica na bacia Amazônica e assim avaliar melhor o efeito da variabilidade climática nas sub-bacias componentes, como o caso do Solimões, e caracterizar os períodos de cheia e estiagem para a melhoria da rede de monitoramento e avaliação de impactos sobre os ambientes locais.

\section{REFERÊNCIAS BIBLIOGRÁFICAS}

ALCÂNTARA, C. R. Linha de instabilidade da Amazônia: estudo de caso e importância das características do perfil do vento na sua formação e desenvolvimento. Ciência \& Natura, v. 33, n. 2, p. 197-226, 2011.

AlenCAR, L. P.; SediYAMA, G. C. S.; WANDERLEY, H. S.; ALMEIDA, T. S.; DELGADO, R. C. Avaliação de Métodos de Estimativa da Evapotranspiração de Referência para três Localidades do Norte de Minas Gerais. Engenharia na Agricultura, v. 19 n. 5, p. 437-449, 2011.

ALVES, E. D. L.; VECCHIA, F. A. S. Analise de Diferentes Métodos de Interpolação para a Precipitação Pluvial no Estado de Goiás. Acta Scientiarum Human and Social Sciences, v. 33, n. 2, p. 193-197, 2011. 
AONASHI, K, J.; AWAKA, M. H.; KOZU, T.; KUBOTA, T.; LIU, G.; SHIGE, S. GSMaP Passive, Microwave Precipitation retrieval algorithm: Algorithm Description and Validation. Journal of Meteorology, v. 87A, p. 119-136, 2009.

ARAÚJO, A. N.; GUETTER, A. K. Validação da Chuva Estimada por Satélite "CMORPH" na Modelagem Hidrológica do Alto Iguaçú. Rev. Bras. de Recursos Hídricos, v. 12, n. 3, p. 189198, 2007.

ARAúJO, G. L.; REIS, E. F.; MARTINS, C. A. S. Desempenho Comparativo de Métodos para Estimativa da Evapotranspiração de Referência. Rev. Bras. de Agricultura Irrigada, v. 5, n. 2, p. 84-95, 2011.

BALTOKOSKI, V.; TAVARES, M.H.F.; MACHADO, R.E. \& OLIVEIRA, M.P. Calibração de modelo para a simulação de vazão e de fósforo total nas sub-bacias dos rios Conrado e Pinheiro - Pato Branco (PR). Rev. Bras. de Ciências do Solo, v. 34, p. 253-261, 2010.

BARROS, V.; DOYLE, M. Precipitation trends in Southern South América to the east of the Andes. Center for Ocean-Land-Atmosphere Studies. Report n० 26, p. 76-80, 1996.

BHATT, C. B.; NAKAMURA, K. Characteristics of monsoon rainfall around the Himalayas revealed by TRMM precipitation radar. Mon. Wea. Rev., v. 133, p. 149-165, 2005.

BINDLISH, R.; BARROS, A. Disaggregation of rainfall for one-way coupling of atmospheric and hydrological models in regions of complex terrain. Global Planet, v. 25, p. 111-132, 2000.

CALHEIROS, A. J. P.; MACHADO, L. A. T. Cloud and rain liquid water statistics in the CHUVA campaign. Atmospheric Research, v. 144, n. SI, p. 126-140, 2014.

CARVALHO, J. R. P.; ASSAD, E. D. Análise espacial da precipitação pluviométrica no Estado de São Paulo: Comparação de métodos de interpolação. Engenharia Agrícola, v. 25, n. 2, p. $377-384$. 2005.

COLLISCHONN, B.; ALLASIA, D.; COLLISCHONN, W.; TUCCI, C. E. M. Desempenho do satélite TRMM na estimativa de precipitação sobre a bacia do Paraguai superior. Rev. Bras. de Cartografia, n. 59, v. 1, p. 93-99, 2007.

COLLISCHONN, B.; COLLISCHONN, W.; TUCCI, C.E.M. Daily hydrological modeling in the Amazon basin using TRMM rainfall estimates, Journal of Hydrology, v. 360, p. 207-216, 2008.

CONSELHO NACIONAL DE RECURSOS HÍDRICOS - CNRH (Brasil). Resolução n 30, de 11 de dezembro de 2002. Diário Oficial da União, Brasília, 19 mar. $2003 a$.

CUARTAS, L. A.; TOMASELlA, J.; NOBRE, A. D.; NOBRE, C. A.; HODNETT, M. G.; WATERLOO, M. J.; MOTA DE OLIVEIRA, S. Distributed hydrological modeling of a micro-scale rainforest watershed in Amazonia: Model evaluation and advances in calibration using the new HAND terrain model. Journal of Hydrology, v. 462-463, p. 15-27, 2012.

DA ROCHA, R. P.; MORALES, C. A.; CUADRA, S. V.; AMBRIZZI, T. Precipitation diurnal cycle and summer climatology assessment over South America: An evaluation of Regional Climate Model version 3 simulations. Journal of Geophysical Research, v. 114, 2009.

DAVIDSON, E. A. et al. The Amazon basin in transition. Nature, v. 481, n. 7381, p. 321-328, 2012.

DEMARIA, M. Linear response of a stratified tropical atmosphere to convective forcing. Journal of the Atmospheric Sciences, v. 42, p. 1944-1959, 1985.

DUAN, Q.; SOROOSHIAN, S.; GUPTA, V. K. Effective and efficient global optimization for conceptual rainfall-runoff models. Water Resour. Res., v. 28, n. 4, p. 1015-1031, 1992. 
FIGUEROA, S. N.; SATYAMURTY, P.; SILVA DIAS, P. L. Simulations of the summer circulation over the South American region with an eta coordinate model. Journal of the Atmospheric Sciences, v. 52 , p. $1573-1584,1995$.

FILHO, H. C. C.; STAINKE, T. E.; STAINKE, V. A. Análise espacial da precipitação pluviométrica na bacia do lago Paranoá: comparação de métodos de interpolação. Revista Geonorte, v. 1, n. 5, p. 336-3455, 2013.

FISCH, G.; MARENGO, J.; NOBRE, C. A. Clima da Amazônia. Climanálise Especial, Edição Comemorativa de 10 anos, p. 24-41, 1996.

GARCEZ, L. N.; ALVAREZ, G. A. Hidrologia. São Paulo: Edgard Blücher, 1988, 291p.

GUILHON, L. G. F.; ROCHA, V. R. Comparação de Métodos de Previsão de Vazões Naturais Afluentes a Aproveitamentos Hidroelétricos. Rev. Bras. de Recursos Hídricos, v. 12, n. 3, p. 1320. 2007.

HALLAK, R.; FILHO, A. J. P. Metodologia para Análise de Desempenho de Simulações de Sistemas Convectivos na Região Metropolitana de São Paulo com o Modelo ARPS: Sensibilidade a Variações com os Esquemas de Advecção e Assimilação de Dados. Rev. Bras. de Meteorologia, v. 26, n. 4, p. 591-608, 2011.

HAMED, A.; KUO-LIN, H.; SOROOSH, S.; BRAITHWAITE, D. K.; KNAPP, K. R.; CECIL, L..; NELSON B. R.; OLIVIER P. Daily Precipitation climate data record from multisatellite observations for hydrological and climate studies. Bulletin of the American Meteorological Society, v. 96, p. 69-83, 2015.

HOU, A. Y.; KAKAR, R. K.; NEECK, STEVEN.; AZARBARZIN, A. A.; KUMMEROW, C. D. ; KOJIMA, M.; OKI, R.; NAKAMURA, KENJI.; IGUCHI, T. The Global Precipitation Measurement Mission. American Meteorological Society, p. 701-722, 2014.

HUFFMAN, G. J.; ADLER, R. F.; MORRISSEY, M.; BOLVIN, D. T.; CURTIS, S.; JOYCE, R.; MCGAVOCK, B.; SUSSKIND, J. Global Precipitation at One-Degree Daily Resolution from Multisatellite Observations. Journal Hydrometeorology, v. 2, p. 36-50, 2001.

HUFFMAN, G. J.; ADLER, R. F.; MORRISSEY, M.; BOLVIN, D. T.; CURTIS, S.; JOYCE, R.; MCGAVOCK, B.; SUSSKIND, J. The TRMM Multisatellite Precipitation Analysis (TMPA): Quasi-global, multiyear, combined-sensor precipitation estimates at fine scales. Journal Hydrometeorology, v. 8 , p. 38-55, 2007

HUFFMAN, G. J.; ADLER, R. F.; RUDOLF, B.; SCHNEIDER, U.; KEEHN P. R. Global precipitation estimates based on a technique for combining satellite-based estimates, rain gauge analysis, and NWP model precipitation information. Journal Climate, v. 8, p. 1284-1295, 1995.

JOYCE, R. J.; XIE, P.; JANOWIAK, J. E. Kalman filter-based CMORPH. Journal Hydrometeorology. v. 12, p. 1547-1563, 2011.

KEENAN, R. J. Climate change impacts and adaptation in forest management: a review. Annals of Forest Science, v. 72, n. 2, p. 145-167, 2014.

KIDD, C.; D. R. KNIVETON, M. C. TODD.; BELLERBY, T. J. Satellite rainfall estimation using combined passive microwave and infrared algorithms. Journal Hydrometeorology. v. 4, p. 1088-1104. 2003.

KIDD, C.; V. LEVIZZANI. Status of Satellite Precipitation Retrievals. Hydrol. Earth Syst. Sci., v. 7, p. 8157-8177, 2011.

KOUSKY, V. E. Atmospheric Circulation Changes associated with Rainfall Anomalies over Tropical Brazil. Monthly Weather Review, v. 113, p. 1951-1957, 1985.

KUBOTA, T.; SHIGE, S.; HASHIZUME, H.; AONASHI, K ; TAKAHASHI, N.; SETO, S.; TAKAYABU, Y. N.; USHIO, T.; NAKAGAWA, K.; IWANAMI, K.; KACHI, M.; OKAMOTO, K. Global precipitation map 
using satellite borne microwave radiometers by the GSMaP project: Production and validation. Geoscience and Remote Sensing, IEEE Transactions, v. 45, p. 2259-2275, 2007.

KULIGOWSKI, R. J. A self-calibrating real-time GOES rainfall algorithm for short-term rainfall estimates. Journal Hydrometeorology, v. 3, p. 112-130. 2002.

KUMMEROW, C.; SIMPSON, J.; THIELE, O.; BAENES, W. The status of the rainfall measuring mission (TRMM) after two years in orbit. Journal of Applied Meteorology, v. 39, p. 1965-1982, 2000.

LAU, W. K. M.; ZHOU, Y. P. Observed recent trends in tropical cyclone rainfall over the North Atlantic and the North Pacific. Journal of Geophysical Research, v. 117, p. 1-13, 2012.

LI, M.; SHAO, Q.; ZHANG, L.; CHIEW, F. H. S. A new regionalization approach and its application to predict flow duration curve in ungauged basins. Journal of Hydrology, v. 389, n. 1-2, p. 137$145,2010$.

MAIDMENT, D. R. Handbook of hydrology. New York: Ed. McGraw-Hill, 1993, 1424 p.

MARENGO, J. A. Long-term trends and cycles in the hydrometeorology of the Amazon basin since the late 1920s. Hydrological Processes, v. 23, n. 22, p. 3236-3244, 2009.

MARENGO, J. A.; SOARES, W. R.; SAULO, C.; NICOLINI, M. Climatology of the Low-Level Jet East of the Andes as Derived from NCEP-NCAR Reanalyses: Characteristics and Temporal Variability. Journal of Climate, v. 17, n. 12, p. 2261-2280, 2004.

MARTINI, A.; VILTARD, N.; ELLIS, S. M.; FONTAINE, E. Ice microphysics retrieval in the convective systems of the Indian Ocean during the CINDY-DYNAMO campaign. Atmosférica Research, $v$. 163, p. $13-23,2015$.

MORUZZI, R. B.; CONCEIÇÃO, F. T.; SARDINHA, D. S.; HONDA, F. P.; NAVARRO, G. R. B. Avaliação de cargas difusas e simulação de autodepuração no córrego da Água Branca, Itirapina (SP). Geociências, v. 31, n. 3, p. 447-458, 2012.

NASH, J. E.; SUTCLIFFE, J. V. River flow forecasting through conceptual models: a discussion of principles. Journal of Hydrology, v. 10, p. 282-290, 1970.

NESBITT, S. W.; ZIPSER, E. J.; CECIL, D. J. A census of precipitation features in the tropics using TRMM: Radar, ice scattering, and lightning observations. Journal. Climate, v. 13, p. 4087-4106, 2000.

NIETO FERREIRA, R. N.; RICKENBACH, T. M.; HERDIES, D. L.; CARVALHO, L. M. V. Variability of South American convective cloud systems and tropospheric circulation during January-March 1998 and 1999. Monthly Weather Review, v. 131, p. 961-973, 2003.

NOBRE, C.A.; SELLERS, P.J.; SHUKLA, J. Amazonian Deforestation and regional climate change. Journal of Climate, v. 4, n. 10, p. 957 - 988, 1991.

NÓBREGA, R.S.; SOUZA, E.P.; GALVÍNCIO, J.D. Análise da estimativa de precipitação do TRMM em uma sub-bacia da Amazônia Ocidental. Revista de Geografia, v. 25, n. 1, p. 6-20, 2008.

PEREIRA FILHO, A. J.; NEGRI, A.; NAKAYAMA, P. T. An inter: comparison of gauge, radar and satellite rainfall in the tropics. 1st International Precipitation Working Group Workshop, CGMS: WMO, Proceedings, p. 275-284, Madrid, Spain, 2002.

REBOITA, M.; GAN, M.; ROCHA, R.; AMBRIZZI, T. Regimes de precipitação na América do Sul: uma revisão bibliográfica. Rev. Bras. de Meteorologia, v. 25, p. 185-204, 2010.

ROZANTE, J. R.; MOREIRA, D. S.; GONÇALVES, L. G. G.; VILA, D. Combining TRMM and surface observation of precipitation: technique and validation over South America. Weather and Forecasting, v. 25, p. 885-894, 2010. 
SALIO, P.; NICOLINI, M.; ZIPSER, J. Mesoscale convective systems over southeastern South American low-level jet. Monthly Weather Review, v. 135, p. 1290-1309, 2007.

SANTI, A. L.; AMADO, T. J. C.; SILVA, V. R.; BASSO, C. J.; DELLA FLORA, L. P.; CHERUBIN, M. R.; EITELWEIN, M. T. Infiltração de água no solo, determinada por diferentes métodos, como indicador do potencial produtivo em dois Latossolos manejados com agricultura de precisão. Interciência, v. 37, p .204-208, 2012.

SANTOS, R. S.; COSTA, L. C.; SEDIYAMA, G. C.; LEAL, B.G.; OLIVEIRA, R. A.; JUSTINO, F. B. Avaliação da relação seca/produtividade agrícola em cenário de mudanças climáticas. Rev. Bras. de Meteorologia, v. 26, p. 313-321, 2011.

SERRÃO, E. A. O.; SANTOS, C. A.; LIMA, A. M. M. Avaliação da seca de 2005 na Amazônia: uma análise da calha do rio Solimões. Estação Científica, v. 4, n. 2, p. 99-109, 2015.

SILVA DIAS, P. L.; SCHUBERT, W. H.; DEMARIA, M. Large-scale response of the tropical atmosphere to transient convection. Journal of the Atmospheric Sciences, v. 40, p. 2689$2707,1983$.

SILVA, P. M. O.; MELLO, C. R.; SILVA, A. M.; COELHO, G. Modelagem da hidrógrafa de cheia em uma bacia hidrográfica da região Alto Rio Grande. Rev. Bras. Eng. Agríc. Ambient. v. 12, n. 3, p. $258-265,2008$.

SOUZA, R. C.; CAMARgo, M. E. Análise e Previsão de Séries Temporais: Os Modelos ARIMA. Rio de Janeiro: Gráfica e Editora Regional, 2004, 187 p.

SOROOSHIAN, S.; HSU, K-L.; GAO, X.; GUPTA, H. V.; IMAM, B.; BRAITHWAITE, D. Evaluation of PERSIANN System Satellite-Based Estimates of Tropical Rainfall. Bulletin of the American Meteorological Society, v. 81, p. 2035-2046, 2000.

STONE, R. J. Improved statistical procedure for the evaluation of solar radiation estimation models. Solar Energy, v. 51, n. 4, p. 289-291, 1993.

TAO, W.-K.; LANG, S.; ZENG, X.; SHIGE. S.; TAKAYABU, Y. Relating convective and stratiform rain to latent heating. Journal Climate, 23, p. 1874-1893, 2010.

THEIL, H. Applied Economic Forecasting. Amsterdam, Netherlands: North-Holland, 1966, 474 p.

TOSHI, M.; TAO, W. K.; MUNCHAK, S. J.; MIRCEA GRECU.; HUFFMAN, G. J. Satellite view of quasiequilibrium states in tropical convection and precipitation microphysics. Geophysical Research Letters, v. 42, p. 1959-1968, 2015.

TRENBERTH, K.; SMITH, L.; QIAN, T.; DAI, A.; FASULLO, F. Estimates of the global water budget and its annual cycle using observational and model data. Journal Hydrometeorology, v. 8, p. 758-769, 2007.

TURK, F. J.; MILLER, S. D. Toward improved characterization of remotely sensed precipitation Regimes with MODIS/AMSR-E blended data techniques: geoscience and remote sensing. IEEE Trans. Geosci. Remote Sens., v. 43, p. 1059-1069, 2005.

ULGEN, K.; HEPBASLI, A. Diffuse solar radiation estimation models for Turkey's big cities. Energy Conversion and Management, v. 50, n. 1, p. 149-156, 2009.

WILLMOTT, C. J.; CKLESON, S. G.; DAVIS, R. E. Statistics for evaluation and comparisons of models. Journal of Geophysical Research, v. 90, n. C5. p. 8995-9005, 1985.

YANG, S.; NESBITT, S. W. Statistical properties of precipitation as observed by the TRMM precipitation radar. Geophysical Research Letters, v. 41, p. 5636-5643, 2014.

YONG, B.; LIU, D.; GOURLEY, J. J.; TIAN, Y.; HUFFMAN, G. J.; REN, L.; HONG, Y. Global view of real-time TRMM multisatellite precipitation analysis: implications for its successor Global 
Precipitation Measurement Mission. Bulletin of the American Meteorological Society, v. 96, n. 2, p. 283-296, 2015.

ZHOU, Y.; LAU, W. K. M.; HUFFMAN, G. J. Mapping TRMM TMPA into Average Recurrence Interval for Monitoring Extreme Precipitation Events. Journal of Applied Meteorology and Climatology, v. 54, p. 979-995, 2015.

ZULUAGA, M. D.; JUNIOR, R. A. H. Extreme Convection of the Near-Equatorial Americas, Africa, and Adjoining Oceans as seen by TRMM. Monthly Weather Review, v. 143, p. 298-316, 2015. 